\title{
Pretreatment with Shuanghe-Tang Extract Attenuates Postischemic Brain Injury and Edema in a Mouse Model of Stroke: An Analysis of Medicinal Herbs Listed in Dongui Bogam
}

\author{
Min Jae Kim, ${ }^{1,2,3}$ Seo-Yeon Lee $\mathbb{D}^{1,2,3}$ Ji Young Hwang, ${ }^{1,2,3}$ Hyunha Kim, ${ }^{1,2,3}$ \\ Ki-Tae Ha $\mathbb{D}^{1,2,3}$ Byung Tae Choi $\mathbb{D}^{1},{ }^{1,2,3}$ Jin Ung Baek ${ }^{(D)}{ }^{4}$ and Hwa Kyoung Shin ${ }^{1}{ }^{1,2,3}$ \\ ${ }^{1}$ Department of Korean Medical Science, School of Korean Medicine, Pusan National University, Yangsan, \\ Gyeongnam 50612, Republic of Korea \\ ${ }^{2}$ Korean Medical Science Research Center for Healthy Aging, Pusan National University, Yangsan, \\ Gyeongnam 50612, Republic of Korea \\ ${ }^{3}$ Graduate Training Program of Korean Medicine for Healthy Aging, Pusan National University, Yangsan, \\ Gyeongnam 50612, Republic of Korea \\ ${ }^{4}$ Division of Humanities and Social Medicine, School of Korean Medicine, Pusan National University, Yangsan, \\ Gyeongnam 50612, Republic of Korea \\ Correspondence should be addressed to Jin Ung Baek; mukjagan@pusan.ac.kr and Hwa Kyoung Shin; julie@pusan.ac.kr
} Received 1 August 2017; Accepted 31 October 2017; Published 11 February 2018

Academic Editor: Angel Catalá

Copyright (C) 2018 Min Jae Kim et al. This is an open access article distributed under the Creative Commons Attribution License, which permits unrestricted use, distribution, and reproduction in any medium, provided the original work is properly cited.

\begin{abstract}
Aim. Although stroke is among the leading causes of death and long-term disability, there are few effective treatments for limiting the severity of neurological sequelae. We evaluated the effects of 29 medicinal herbs listed in the Pung chapter of the 17th century Korean medical text Dongui Bogam on stroke symptoms in a mouse model of cerebral ischemia. Methods. Focal cerebral ischemia was induced via photothrombosis. Infarct volume, brain edema, and neurological deficits were evaluated. Immunofluorescence staining for tight junction proteins and aquaporin 4 (AQP4) was performed following ischemic injury. Results. Based on our initial findings, we examined the effects of two prescriptions in which the candidate herbs comprised more than $60 \%$ of the total formula: Shuanghe-tang and Zengsunsiwu-tang. Pretreatment with Shuanghe-tang significantly reduced infarct volume, decreased blood-brain barrier (BBB) breakdown, attenuated edema, and improved neurological and motor functions in a dosedependent manner $(30,100$, and $300 \mathrm{mg} / \mathrm{kg})$, while no such effects were observed in mice pretreated with Zengsunsiwu-tang. Immunohistochemical analysis revealed significant increases in ipsilateral occludin and zonula occludens 1 (ZO-1) expression in Shuanghe-tang-pretreated mice, as well as increased AQP4 immunofluorescence. Conclusions. These results indicate that Shuanghe-tang may protect against brain injury and promote recovery of neurological function following ischemia.
\end{abstract}

\section{Introduction}

Stroke is among the leading causes of death and long-term disability worldwide, affecting an estimated 15 million people each year. According to a 2002 report by the World Health Organization, approximately $30 \%$ of patients with stroke experience long-term sequelae and reduced quality of life due to stroke-related disability $[1,2]$. While tissue plasminogen activator (tPA) has been approved by the FDA for the treatment of ischemic injury, tPA treatment must be initiated within 4.5 hours of occlusion [3]. Due to the substantial limitations inherent to tPA treatment, researchers have aimed to identify alternative pharmacological options for combating ischemic disease. Among such options, the pharmaceutical industry has recently begun to investigate the potential of herbs historically utilized in traditional medicine settings [4].

Traditional medicines are composed of natural products, which represent a promising source of new ingredients for the development of conventional medicines. Indeed, 
researchers have begun to consult the traditional medicine literature in the search for novel therapeutic strategies $[5,6]$. Compiled by the Korean royal physician Heo Jun during the 17th century, the Dongui Bogam contains records regarding a number of prescriptions and systematic screening methods that have been applied to both experimental and clinical settings $[7,8]$. Researchers have suggested that the Pung chapter of the Dongui Bogam contains valuable information regarding herbal combinations that may aid in the development of novel stroke treatments [9].

A number of pathophysiological events occur following stroke, including energy failure, glutamate excitotoxicity, oxidative stress, leukocyte infiltration, inflammation, breakdown of the blood-brain barrier (BBB), and edema [10]. The BBB maintains the homeostasis of the central nervous system (CNS), which regulates material exchange between the blood and the brain parenchyma [11]. The primary barrier of the $\mathrm{BBB}$ is formed by microvascular endothelial cells, which are connected to one another via tight junctions. Research has indicated that specific tight junction proteins are expressed in these regions and that the endothelial cells of the $\mathrm{BBB}$ are tightly wrapped by astrocytic endfeet [11, 12]. Following ischemic stroke, the permeability of the BBB increases, allowing circulating immune cells to infiltrate to the brain [13]. In addition, brain edema promotes expansion of the infarct area [14]. Previous studies have revealed that compromised $\mathrm{BBB}$ integrity is associated with decreased levels of tight junction proteins such as zonula occludens 1 (ZO-1) and occludin in experimental models of neurological disease [15]. Aquaporin 4 (AQP4) is the most abundant type of water channel and is mainly expressed in the astrocytic endfeet that surround capillaries in the cortex and striatum [16]. While several lines of evidence have suggested that $\mathrm{AQP} 4$ plays important roles in maintaining $\mathrm{BBB}$ integrity and edema, research regarding the effects of AQP4 on $\mathrm{BBB}$ injury and cerebral edema remains controversial [17-19].

Thus, the prevention of BBB breakdown and edema is critical for advancing research regarding treatment options for ischemic stroke. In the present study, we examined the effects of 29 medicinal herbs listed in the Dongui Bogam on stroke symptoms in a mouse model photothrombotic cortical ischemia.

\section{Materials and Methods}

2.1. Selection of Medicinal Herbs for the Treatment of Stroke. The medicinal herbs examined in the present study were selected by searching all prescriptions for the treatment of stroke (PTSs) listed in the Pung chapter of the Dongui Bogam via text mining analysis. Among the 92 PTSs identified, we selected medicinal herbs with the highest repeat frequencies in the Korean medical literature (Figure 1). Overlapping combinations of herbs frequently reported in the literature were also chosen as candidates. We then determined the dose of each medicinal herb within a prescription as a percentage of the total prescription. Those with a higher proportion of the medicinal herb were classified as main prescriptions [9]. Only those herbs within the top $80 \%$ as a cumulative percentage of each prescription were selected, resulting in a total of
33 herbs. Among these, we further excluded those derived from animals or minerals, resulting in a final total of 29 medicinal herbs (Figure 1). The 29 herbs were extracted via freeze drying, following which the yield of each extract was calculated (Table 1). PKM03-28 and PKM03-29 were excluded from further investigation due to their toxicity and liquid formula, respectively (data not shown).

2.2. Preparation of Herb Extracts. The 29 selected herbs were purchased from Hwalim Natural Drug (Busan, Korea). Extracts were prepared by adding an amount of $70 \%$ ethanol equal to 10 times the weight of the herb. Each herb was extracted via sonication, which was performed three times for each sample over an interval of $1 \mathrm{~h}$, following which samples were filtered twice and concentrated using an evaporator equipped with a decompression device. The yield of herb extracts was calculated after freeze drying (Supplementary Figure S1A). Extracts of Shuanghe-tang and Zengsunsiwutang were by Pusan National University Korean Medicine Hospital. Shuanghe-tang or Zengsunsiwu-tang was boiled in $1.2 \mathrm{~L}$ of water for $3 \mathrm{~h}$ and then extracted as described above (Supplementary Figure S1B).

2.3. Animal Experiments. Male C57BL/6 mice were obtained from Doyeol Biotech (Seoul, Korea). Mice were housed under a $12: 12 \mathrm{~h}$ light/dark cycle and allowed ad libitum access to food and water. All animal protocols were implemented in accordance with Pusan National University guidelines for the care and use of laboratory animals and had been approved by the Institutional Review Board of Pusan National University (PNU-2016-1064). Mice were orally administered $0.15 \mathrm{ml}$ of each herb extract at the appropriate concentration once per day for 2 days, as well as $1 \mathrm{~h}$ prior to focal cerebral ischemic injury (total of three treatments). Extracts of Shuanghe-tang or Zengsunsiwutang were orally administered to mice once per day for 4 days, as well as $1 \mathrm{~h}$ prior to focal cerebral ischemic injury (total of five treatments).

2.4. Induction of Focal Cerebral Ischemia. Focal cerebral ischemia was induced via photothrombosis as previously described [20]. Briefly, mice were anesthetized with $2 \%$ isoflurane in $\mathrm{O}_{2}(20 \%)$ and $\mathrm{N}_{2} \mathrm{O}(80 \%)$, following which they received an intraperitoneal (i.p.) injection of rose bengal (Sigma-Aldrich, St. Louis, MO; $0.1 \mathrm{ml}$ of $10 \mathrm{mg} / \mathrm{ml}$ in $0.9 \%$ saline) $5 \mathrm{~min}$ prior to illumination. Mice were fixed on a Stereotaxic Frame (David Kopf Instruments, Tujunga, CA), following which the brain was exposed. A fiber optic bundle containing a KL 1500 LED cold light source (Carl Zeiss, Jena, Germany) was positioned onto the sensorimotor cortex of exposed brain (2.4 $\mathrm{mm}$ lateral from the bregma) and illuminated for 15 mins. The scalp was sutured after illumination, at which time the mice were allowed to recover under a heating lamp and returned to their home cages. Body temperature was maintained at $37.5^{\circ} \mathrm{C}$ during surgery using a heating pad (Harvard Apparatus, Holliston, MA).

2.5. Infarct Volume and Edema. Brains were harvested $24 \mathrm{~h}$ after ischemic injury, and infarct size was determined via 2,3,5-triphenyltetrazolium chloride (TTC) staining of 


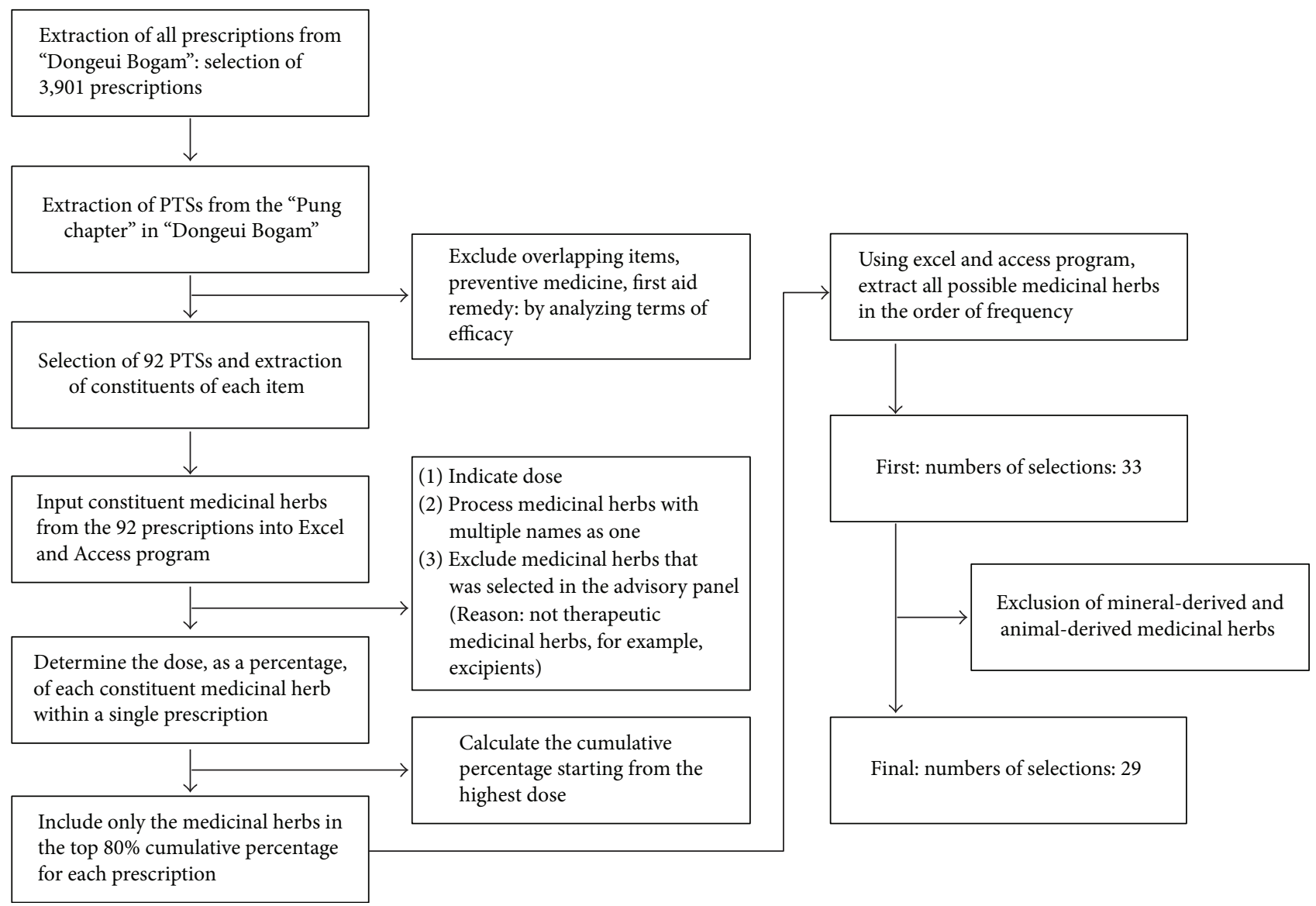

Figure 1: Selection of medicinal herbs for stroke treatment from 92 prescriptions listed in Dongui Bogam.

$2 \mathrm{~mm}$ thick brain sections. Infarct size was quantified using i-Solution software (Image \& Microscope Technology, Vancouver, Canada). Measurements of direct infarct volume included areas of the ipsilateral side that had sustained direct damage. Indirect infarct volume was calculated according to the following formula: contralateral hemisphere $\left(\mathrm{mm}^{3}\right)-$ undamaged ipsilateral hemisphere $\left(\mathrm{mm}^{3}\right)$. Edema was calculated by subtracting direct infarct volume from indirect infarct volume.

2.6. Neurological Score. Neurological deficits were evaluated $24 \mathrm{~h}$ after ischemic injury using the following scoring system: $1=$ turning in direction of ipsilateral (nondamaged) side when held by the tail; $2=$ turning in direction of contralateral (damaged) side and difficulty bearing weight; $3=$ unable to bear weight on the contralateral side; $4=$ no spontaneous movement [21].

2.7. Rota-Rod Test. Locomotor function was examined using the rota-rod test, based on the average latency until falling from the spinning rod (Panlab S.L.U., Barcelona, Spain). Mice underwent pretraining for adaptation trials, following which they were placed on the rotating rod at a speed of $18 \mathrm{rpm}$ for $3 \mathrm{~min}$. Each mouse underwent five trials.

2.8. Wire Grip. A wire grip test was used to evaluate the vestibular motor function of mice $24 \mathrm{~h}$ after focal cerebral ischemia. Each mouse was suspended on a metal wire and forced to hang using both forepaws. Wire grip was scored as follows: $1=$ not holding onto the wire; 2 =holding onto the wire using both forepaws and hindpaws but not the tail; 3 = holding onto the wire using both forepaws and hindpaws as well as the tail, without movement; $4=$ moving on the wire using both forepaws, both hindpaws, and tail; and $5=$ moving well on the wire.

2.9. Determination of Evans Blue Leakage. Evans Blue (2\% in saline, $4 \mathrm{ml} / \mathrm{kg}$; Sigma-Aldrich) was injected into the tail vein immediately following photothrombotic ischemia. Twentyfour hours after injection, mice were anesthetized and transcardially perfused with PBS. Brains were removed, following which the cortical area of each hemisphere was separated, weighed, and homogenized in $400 \mu \mathrm{l}$ of N,N-dimethylformamide (Sigma-Aldrich, St. Louis, MO, USA). Following incubation overnight at $55^{\circ} \mathrm{C}$, samples were centrifuged at $13,000 \mathrm{rpm}$ for $20 \mathrm{mins}$. The absorbance of supernatant was measured at $620 \mathrm{~nm}$ via spectrophotometry, while Evans Blue extravasation ( $\mu \mathrm{g} / \mathrm{g}$ of brain tissue) was quantified using a standard curve [22].

2.10. Immunofluorescence Staining for Tight Junction Proteins. Mice were perfused with cold PBS followed by $4 \%$ paraformaldehyde $24 \mathrm{~h}$ after focal cerebral ischemia. Immediately thereafter, brains were harvested and further fixed 
TABle 1: List of medicinal herbs for the treatment of stroke selected from Dongui Bogam.

\begin{tabular}{|c|c|c|c|c|}
\hline Family & Scientific name & Portion used & Yield (\%) & Sample number \\
\hline Labiatae & Schizonepeta tenuifolia Briquet & Spike & 3.34 & PKM03-01 \\
\hline Polyporaceae & Poria cocos Wolf & Sclerotium & 0.434 & PKM03-02 \\
\hline Leguminosae & Pueraria lobata Ohwi & Root & 11.52 & PKM03-03 \\
\hline Ephedraceae & Ephedra sinica Stapf & Aerial part & 3.46 & PKM03-04 \\
\hline Rutaceae & Citrus unshiu Markovich & Peel & 12.88 & PKM03-05 \\
\hline Umbelliferae & Saposhnikovia divaricata Schiskin & Root & 9.66 & PKM03-06 \\
\hline Leguminosae & Astragalus membranaceus Bunge & Root & 12.84 & PKM03-07 \\
\hline Ranunculaceae & Cimicifuga heracleifolia Komarov & Rhizome & 12.34 & PKM03-08 \\
\hline Compositae & Atractylodes lancea DC & Rhizome & 16.08 & PKM03-09 \\
\hline Araceae & Arisaema amurense Maximowicz & Tuber & 2.9 & PKM03-10 \\
\hline Paeoniaceae & Paeonia lactiflora Pallas & Root & 7.713 & PKM03-11 \\
\hline Umbelliferae & Angelica gigas Nakai & Root & 15.013 & PKM03-12 \\
\hline Scrophulariaceae & Rehmannia glutinosa Libosch. var. purpurea Mak & Tuber & 20.76 & PKM03-13 \\
\hline Umbelliferae & Ostericum koreanum Maximowicz & Rhizome, root & 12.14 & PKM03-14 \\
\hline Umbelliferae & Cnidium officinale Makino & Rhizome & 9.76 & PKM03-15 \\
\hline Lauraceae & Cinnamomum cassia Blume & Bark & 4.566 & PKM03-16 \\
\hline Polygonaceae & Rheum palmatum Linne & Rhizome, root & 11.77 & PKM03-17 \\
\hline Lauraceae & Lindera strychnifolia Villars & Root & 7.513 & PKM03-18 \\
\hline Rutaceae & Poncirus trifoliata Rafinesque & Fruit & 9.906 & PKM03-19 \\
\hline Ranunculaceae & Aconitum koreanum Raymond & Tuberous root & 19.006 & PKM03-20 \\
\hline Campanulaceae & Platycodon grandiflorum A. De Candolle & Root & 13.33 & PKM03-21 \\
\hline Araliaceae & Aralia continentalis Kitagawa & Root & 7.393 & PKM03-22 \\
\hline Araliaceae & Panax ginseng C. A. Meyer & Root & 10.52 & PKM03-23 \\
\hline Zingiberaceae & Zingiber officinale Roscoe & Rhizome & 7.06 & PKM03-24 \\
\hline Orchidaceae & Gastrodia elata Blume & Tuber & 7.233 & PKM03-25 \\
\hline Labiatae & Scutellaria baicalensis Georgi & Root & 10.606 & PKM03-26 \\
\hline Leguminosae & Glycyrrhiza glabra Linne & Rhizome, root & 16.15 & PKM03-27 \\
\hline
\end{tabular}

for $24 \mathrm{~h}$ in $4 \%$ paraformaldehyde, following which they were cryoprotected in $30 \%$ sucrose for $72 \mathrm{~h}$ at $4^{\circ} \mathrm{C}$. Each brain was frozen in optical cutting temperature (OCT) compound (Sakura Finetek, Torrance, CA) and stored at $-80^{\circ} \mathrm{C}$ until analysis. The frozen brains were sectioned (thickness: $20 \mu \mathrm{m}$ ) using a CM 3050 cryostat (Leica Microsystems, Wetzlar, Germany). Brain sections were immunostained with anti-ZO-1 (1:100), antioccludin (1:100, Invitrogen Corporation, Carlsbad, CA), anti-CD-31(1:100, BD Bioscience), and anti-AQP4 (1:100, Millipore) overnight at $4^{\circ} \mathrm{C}$, following which they were incubated with Alexa 488 or Alexa 594-conjugated secondary antibodies (1:500, Life Technologies) for $2 \mathrm{~h}$ in total darkness. DAPI (molecular probe) was used for nuclei staining. Fluorescence images were captured using a Zeiss LSM 700 laser scanning confocal device (Carl Zeiss, Jena, Germany) and Slide Scanner Axio Scan.Z1 (Carl Zeiss, Jena, Germany). The images are quantified using Metamorph Microscopy Automation and Image Analysis Software (Molecular Devices, USA) and i-Solution software (Image \& Microscope Technology, Vancouver, Canada).

2.11. Statistical Analysis. Data are represented as the mean \pm SEM. Comparisons of mean values between two groups were performed using Student's $t$-tests. The level of statistical significance was set at $p<0.05$.

\section{Results}

3.1. Pretreatment Effects of Medicinal Herbs on Ischemic Brain Injury. We evaluated the pretreatment effects of selected herbs on symptoms of focal cerebral ischemia. Mice were orally administered 100 and $500 \mathrm{mg} / \mathrm{kg}$ of each extract once a day for 2 days prior to ischemic injury, as well as $1 \mathrm{~h}$ prior to the procedure. Twenty-four hours after ischemic insult, the locomotor function of mice was evaluated using the rota-rod test (Figure 2(a)). Our results indicated that 12 herb extracts significantly reduced direct infarct volume (PKM03-02, PKM03-04, PKM03-05, PKM03-07, PKM03-11, PKM03-12, PKM03-16, PKM03-19, PKM0320, PKM03-22, PKM03-24, and PKM03-27), relative to that observed in the PBS-treated control group (Figure 2(b)). Furthermore, pretreatment with eight candidate herbs (PKM03-03, PKM03-05, PKM03-14, PKM03-15, PKM0317, PKM03-18, PKM03-21, and PKM03-27) significantly decreased levels of brain edema relative to those observed in the control group (Figure 2(c)). Functional motor outcomes following ischemic injury were significantly improved 


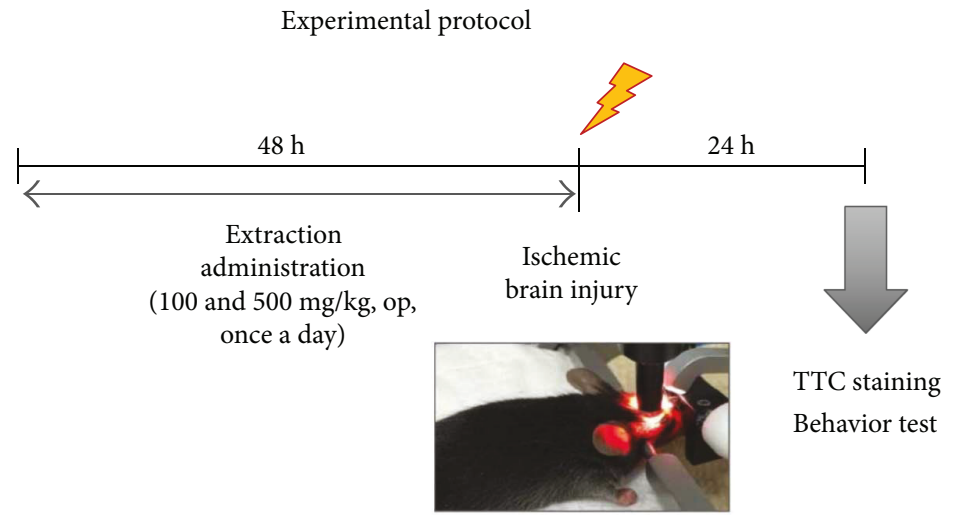

(a)

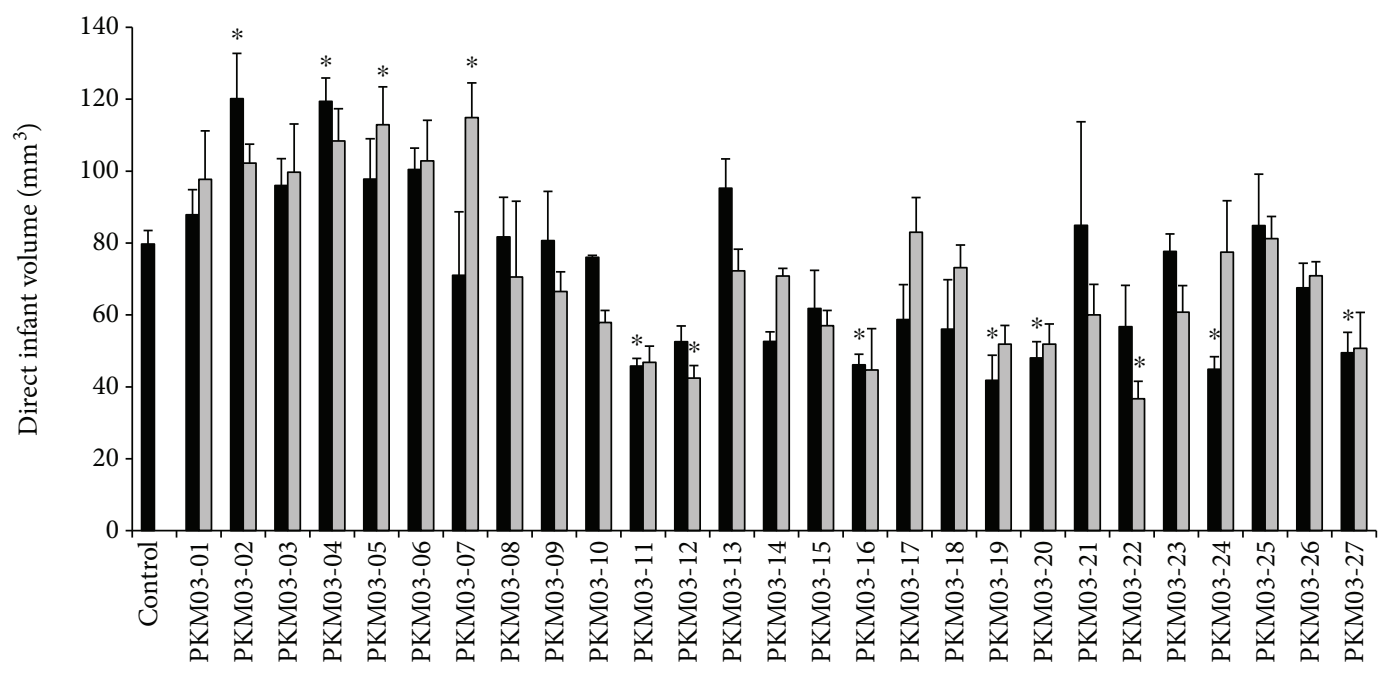

- $100 \mathrm{mg} / \mathrm{kg}$

ㄷ $500 \mathrm{mg} / \mathrm{kg}$

(b)



(c)

Figure 2: Continued. 


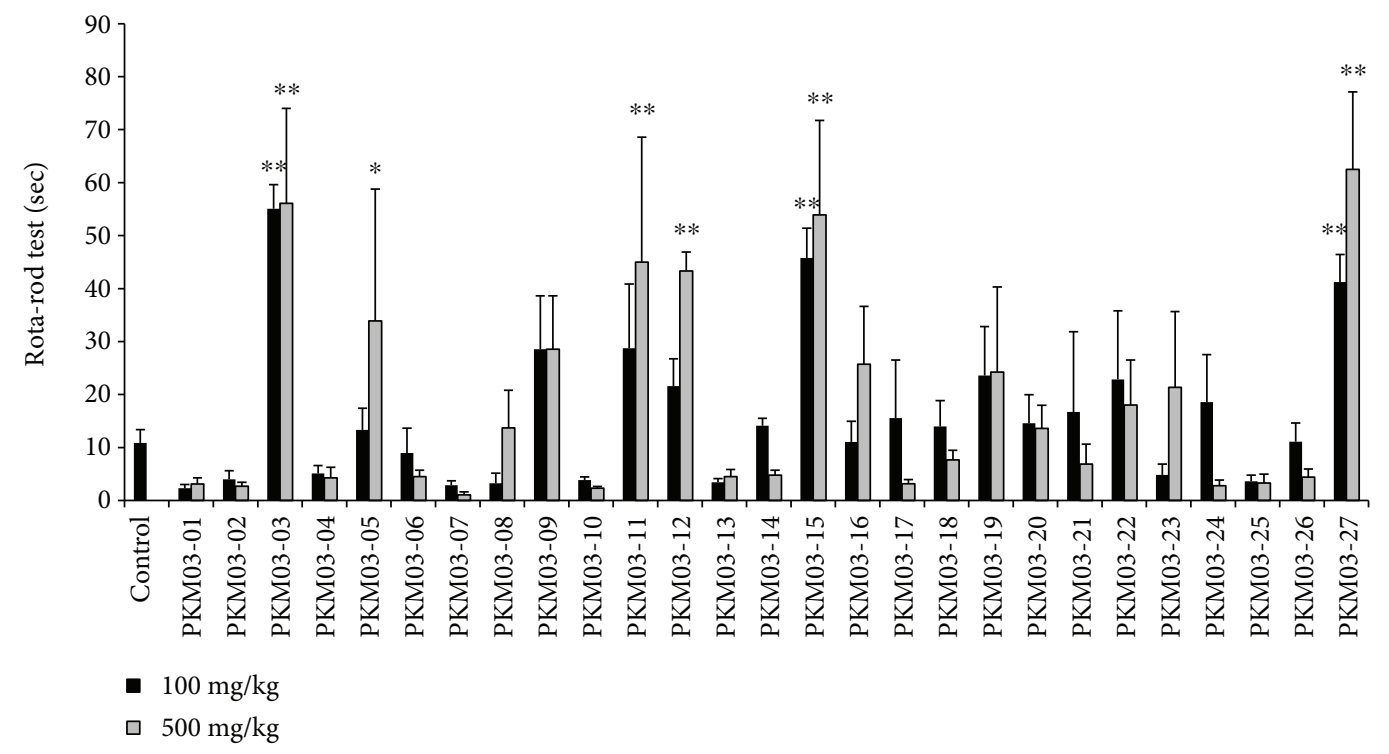

(d)

Figure 2: Effects of medicinal herbs on ischemic brain injury following focal cerebral ischemia. (a) Experimental protocol. Mice were pretreated via oral administration of 100 or $500 \mathrm{mg} / \mathrm{kg}$ of medicinal herb extracts $(n=3-4$ each) or PBS (control group, $n=56)$ for 2 days prior to focal cerebral ischemia, as well as $1 \mathrm{~h}$ prior to the procedure. (b, c) Direct infarct volume (b) and edema (c) were quantified via TTC staining $24 \mathrm{~h}$ after injury. ${ }^{*} p<0.05$ versus control group. (d) The rota-rod test was used to examine locomotor function. The average latency time over five trials was recorded for each mouse. ${ }^{*} p<0.05,{ }^{* *} p<0.01$ versus control group.

in mice pretreated with one of the following six candidate herbs: PKM03-03, PKM03-05, PKM03-11, PKM03-12, PKM03-15, and PKM03-27 (Figure 2(d)). Based on our findings, we chose to further examine five herbs demonstrating significant effects in two of the three analyses (infarct volume, edema, and rota-rod test): PKM03-03, PKM03-11, PKM0312, PKM03-15, and PKM03-27.

3.2. Selection of Formulas Containing the Candidate Herbs. Following selection of the five candidate herbs (PKM03-03, PKM03-11, PKM03-12, PKM03-15, and PKM03-27), we searched the traditional Korean medical literature for formulas containing these herbs, based on the following criteria: (1) The candidate herbs are the main components of the traditional Korean medicine formula; (2) the formula includes at least four of the five candidate herbs; (3) the volume of these four herbs comprise over $60 \%$ of the total volume of the combined formula. Based on these criteria, we selected Shuanghe-tang and Zengsunsiwu-tang (Table 2). Shuanghe-tang and Zengsunsiwu-tang were extracted via freeze drying, following which we investigated their effects on ischemic injury (Supplementary Figure S1).

3.3. Effects of Shuanghe-Tang and Zengsunsiwu-Tang on Ischemic Brain Injury. Mice underwent oral administration of Shuanghe-tang $(500 \mathrm{mg} / \mathrm{kg})$ or Zengunsiweu-tang $(500 \mathrm{mg} / \mathrm{kg})$ once per day for 4 days prior to the induction of ischemia, as well as $1 \mathrm{~h}$ prior to the procedure (Figure 3(a)). Our findings indicated that oral administration of Shuanghe-tang extract significantly reduced the infarct area (Figure 3(b)), direct infarct volume, and extent of edema, relative to values observed in the control group
TABle 2: Composition of Shuanghe-tang and Zengsunsiwu-tang.

\begin{tabular}{lcc}
\hline Family & Scientific name & $\begin{array}{c}\text { Amount } \\
(\mathrm{g})\end{array}$ \\
\hline $\begin{array}{l}\text { Shuanghe-tang } \\
\text { Paeoniaceae }\end{array}$ & Paeonia lactiflora Pallas \\
Leguminosae & Astragalus membranaceus Bunge & 10 \\
Scrophulariaceae & Liboschitz var. purpurea Makino & 4 \\
Umbelliferae & Angelica gigas Nakai & 4 \\
Umbelliferae & Cnidium officinale Makino & 4 \\
Lauraceae & Cinnamomum cassia Blume & 3 \\
Leguminosae & Glycyrrhiza glabra Linne & 3 \\
& Total & 32 \\
\hline Zengsunsiwu-tang & Paeonia lactiflora Pallas & 5 \\
Paeoniaceae & Angelica gigas Nakai & 5 \\
Umbelliferae & Cnidium officinale Makino & 5 \\
Umbelliferae & Panax ginseng C. A. Meyer & 2 \\
Araliaceae & Zingiber officinale Roscoe & 2 \\
Zingiberaceae & Glycyrrhiza glabra Linne & 2 \\
Leguminosae & Total & 21 \\
\hline
\end{tabular}

(Figures 3(c) and 3(d)). Moreover, Shuanghe-tang treatment significantly reduced the severity of neurologic deficits (Figure 3(e)) and improved functional motor ability (Figure 3(f)) relative to findings observed in control mice. In contrast, no significant effects of Zengsunsiwu-tang treatment were observed with regard to infarct volume, edema, neurological deficits, or motor function. We also examined 


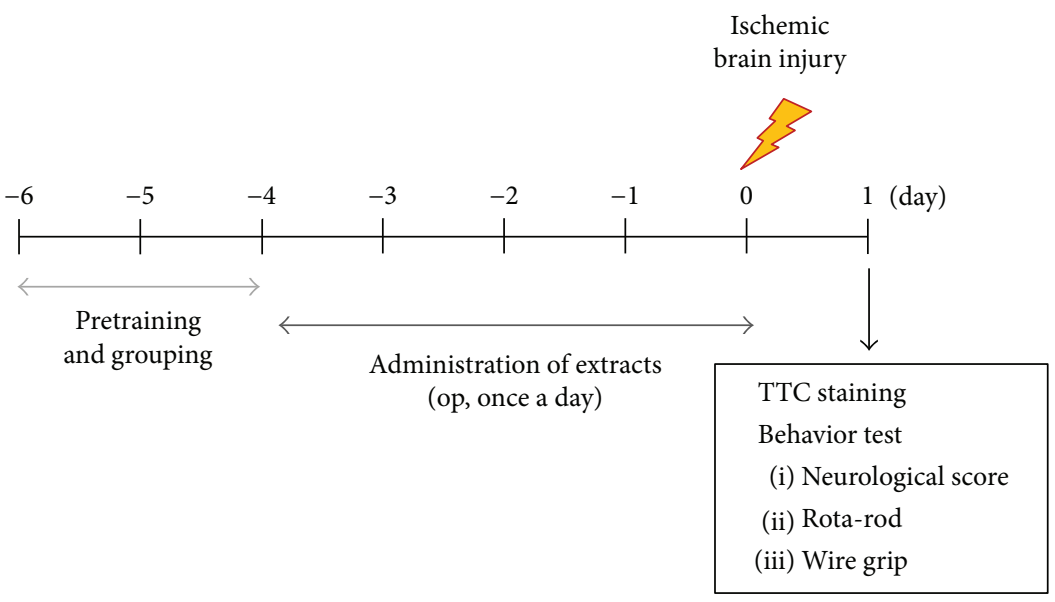

(a)

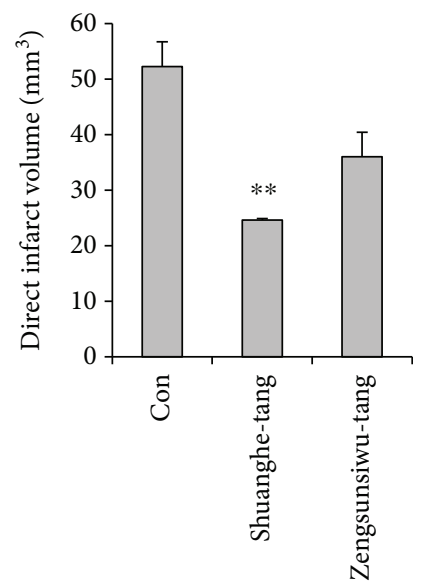

(c)

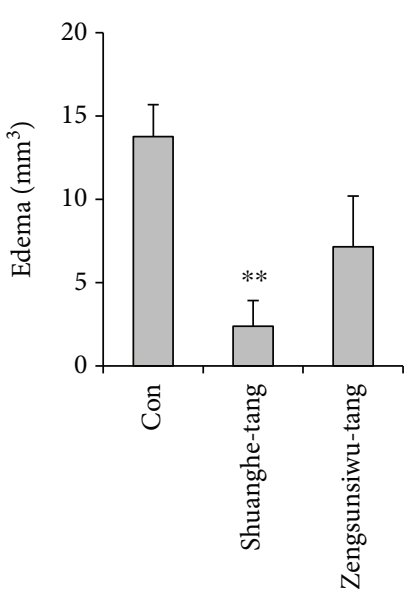

(d)

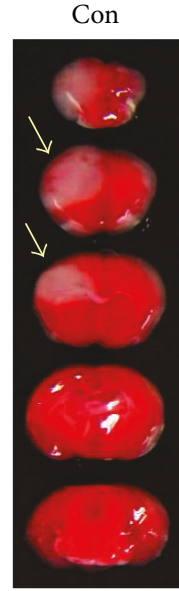

Shuanghe-tang Zengsunsiwu-tang
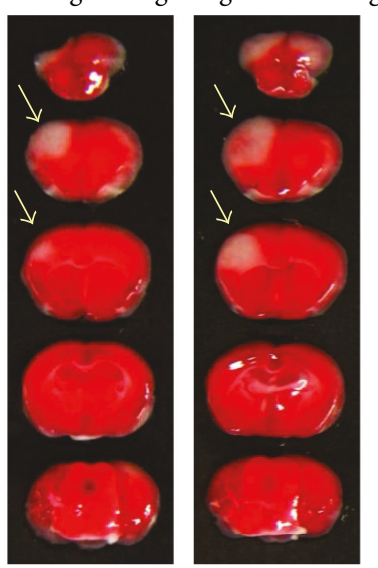

(b)

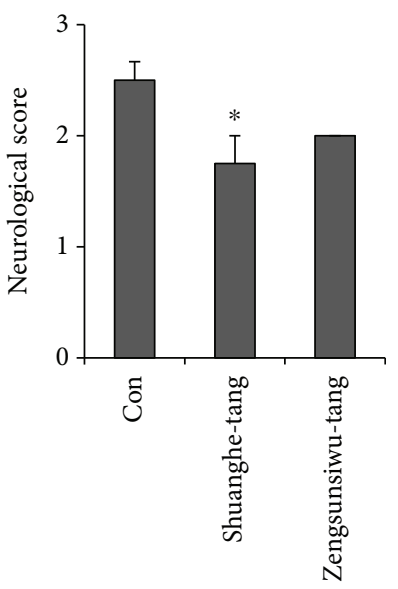

(e)

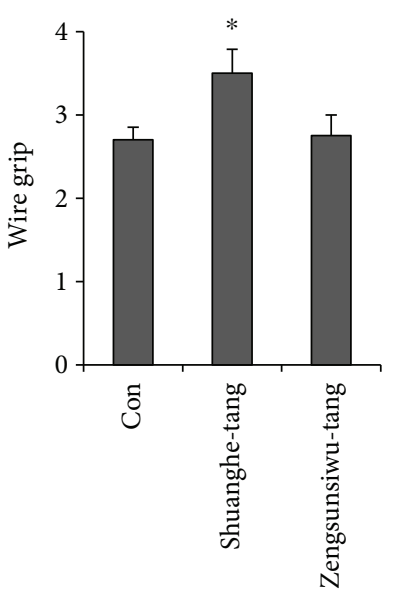

(f)

Figure 3: Effects of Shuanghe-tang and Zengsunsiwu-tang on brain function and behavior following ischemic brain injury. (a) Mice were pretreated via oral administration of $500 \mathrm{mg} / \mathrm{kg}$ of Shuangh-tang or Zengsunsiwu-tang $(n=4)$ or PBS (control group, $n=9)$ once per day for 4 days prior to ischemic injury, as well as $1 \mathrm{~h}$ prior to the procedure. Twenty-four hours after ischemic brain injury, the mouse brains were harvested and stained with 2\% TTC solution. (b) Representative photographs of brain sections stained with TTC. White region (arrows) indicates the infarct area. (c, d) Quantification graphs of direct infarct volume (c) and edema (d). ${ }^{* *} p<0.01$ versus control group. (e, f) Neurological score (e) and wire grip tests (f) were performed to evaluate functional outcomes. ${ }^{*} p<0.05$ versus control group.

the posttreatment effect of Shuanghe-tang following ischemic injury (Supplementary Figure S2). Posttreatment with Shuanghe-tang did not decrease the level of infarct volume and brain edema relative to the control group. Neurological deficits and functional motor outcomes following ischemic injury were not improved in mice treated with Shuanghe-tang.

\subsection{Dose-Dependent Effects of Shuanghe-Tang on Ischemic} Brain Injury. To determine whether Shuanghe-tang exerts dose-dependent effects in a mouse model of ischemic brain injury, mice were pretreated with 30,100 , or $300 \mathrm{mg} / \mathrm{kg}$ of Shuanghe-tang (Figure 4). Our findings indicated that Shuanghe-tang produced dose-dependent reductions in direct infarct and edema volume. The most significant reductions in infarct volume and edema were observed in mice pretreated with $300 \mathrm{mg} / \mathrm{kg}$ of Shuanghe-tang extract (Figures 4(a)-4(c)). We further observed dose-dependent effects of Shuanghe-tang pretreatment on neurologic deficits, locomotor function, and vestibular motor function. Significant improvements in functional outcomes were observed in mice pretreated with $300 \mathrm{mg} / \mathrm{kg}$ of Shuanghe-tang extract, relative to those observed in the control group (Figures 4(d)-4(f)).

3.5. Effects of Shuanghe-Tang on BBB Disruption. Because Shuanghe-tang reduced ischemic brain edema, we evaluated the effect of Shuanghe-tang on the BBB breakdown after ischemia. Evans Blue solution injected to mice extravasates through damaged BBB (Figures 5(a) and 5(b)). After administration of $300 \mathrm{mg} / \mathrm{kg}$ Shuanghe-tang, BBB disruption induced by photothrombotic ischemia was significantly reduced, relative to that observed in the control group (Figures 5(a) and 5(b)). We examined the effect of Shuanghe-tang $(300 \mathrm{mg} / \mathrm{kg})$ on the tight junction proteins for BBB maintenance (Figure 6). Immunofluorescence staining revealed the increased levels of $\mathrm{ZO}-1$ and occludin even following cerebral ischemia, suggesting that Shuanghe-tang 

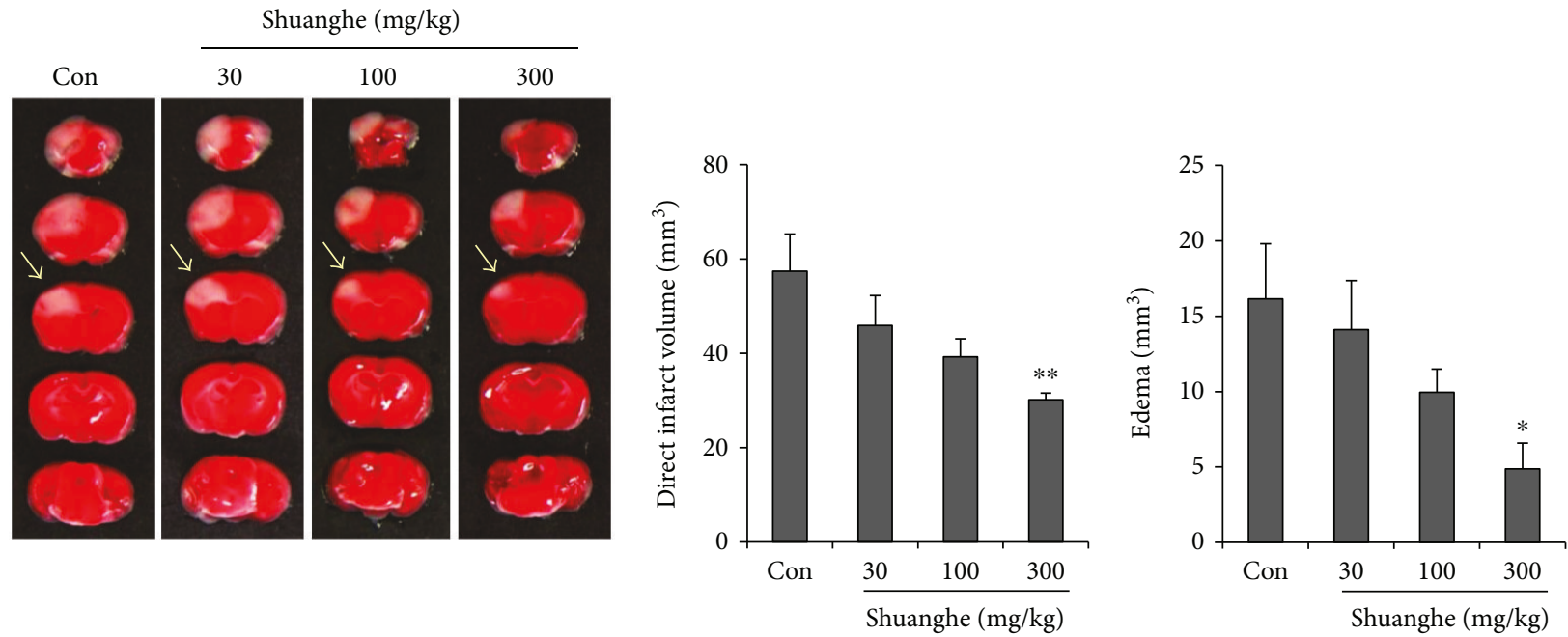

(a)

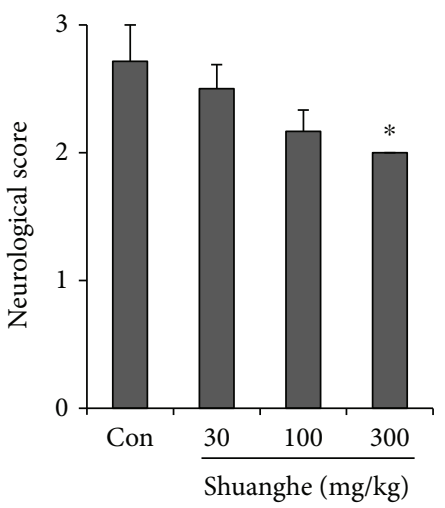

(d) (b)

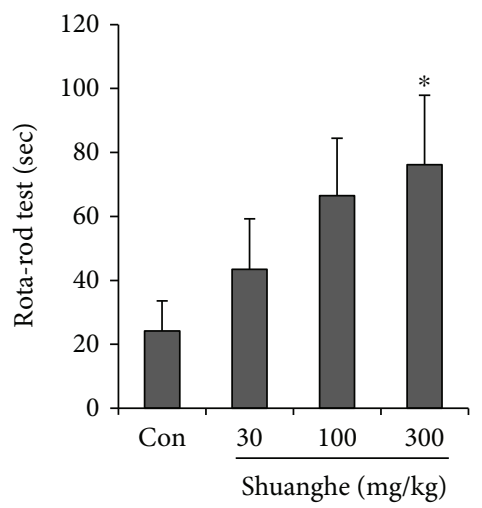

(e) (c)

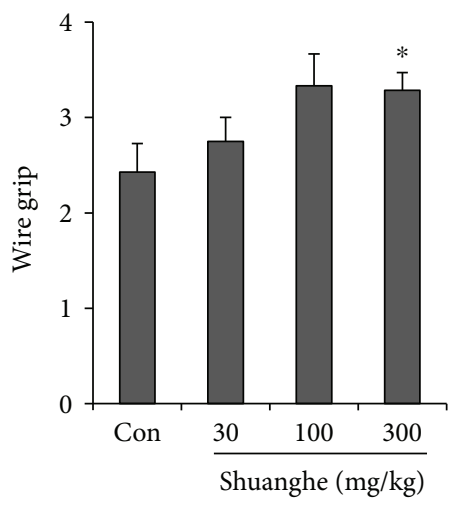

(f)

Figure 4: Dose-dependent effects of Shuanghe-tang on ischemic brain injury. Mice were pretreated via oral administration of 30, 100, or $300 \mathrm{mg} / \mathrm{kg}$ of Shuanghe-tang ( $n=6-8$ each) or PBS (control group, $n=7$ ) once per day for 4 days prior to ischemic insult, as well as $1 \mathrm{~h}$ prior to the procedure. (a) Representative photographs of coronal brain sections stained with TTC. White region (arrows) indicates the infarct area. (b, c) Quantification of direct infarct volume (b) and edema (c) 24 h postischemia. ${ }^{*} p<0.05,{ }^{* *} p<0.01$ versus control group. $(\mathrm{d}-\mathrm{f})$ Neurological score (d), rota-rod (e), and wire grip (f) results were evaluated to assess recovery of neurologic deficit, locomotor function, and vestibular motor function after ischemic injury. ${ }^{*} p<0.05$ versus control group.

reduces $\mathrm{BBB}$ disruption via increases in the level of tight junction proteins.

We then examined the effects of Shuanghe-tang treatment on levels of the main water channel protein, AQP4. Focal cerebral ischemia showed the low level expression of AQP4 in the peri-infarct region (Figures 7(a)-7(c)). However, mice pretreated with Shuanghe-tang exhibited significant increases in AQP-4 expression in the perivascular region (Figures $7(\mathrm{c})$ and $7(\mathrm{~d})$ ). These findings indicate that AQP4 reduction contributes to BBB disruption and the expansion of brain edema and that Shuanghetang extract may protect against secondary injury following ischemia.

\section{Discussion}

In the present study, we evaluated the effects of 27 medicinal herbs selected via text mining analysis of the Korean medical text Dongui Bogam in a mouse model of photothrombotic stroke. Based on initial results regarding infarct volume, edema, and rota-rod performance, we further evaluated the effects of five candidate herbs (PKM03-03, PKM03-11, PKM03-12, PKM03-15, and PKM03-27) in our mouse model of ischemic brain injury (Figure 2).

In traditional Korean medicine, Pueraria lobata (PKM03-03) has been used to treat fever, headache, and cardiovascular diseases such as hypertension [23], while Paeonia lactiflora (PKM03-11) has been used to promote blood circulation and relieve smooth muscle spasms [23]. In addition, Angelica gigas (PKM03-12) has been used to treat anemia in women due to its reported effects on hematogenesis [24]. Cnidium officinale (PKM03-15) has been used to treat menstrual problems as well as pain associated with rheumatic arthralgia [25]. Glycyrrhiza glabra (PKM03-27), one of the most commonly utilized herbs in Korean medicine, has been used in the treatment of renovascular and cardiovascular 


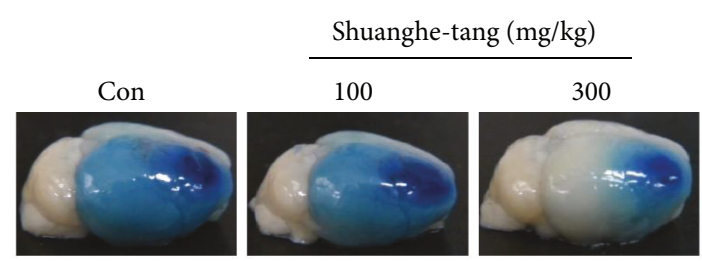

(a)

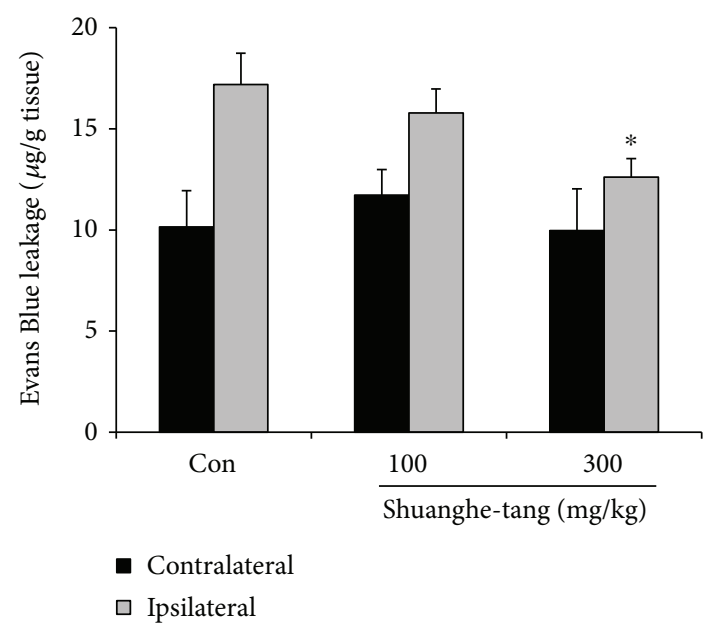

(b)

FIgURE 5: Effects of Shuanghe-tang on BBB disruption after ischemic brain injury. Mice were pretreated via oral administration of 100 or $300 \mathrm{mg} / \mathrm{kg}$ of Shuanghe-tang $(n=10)$ or PBS (control group, $n=10$ ) once per day for 4 days prior to ischemic insult, as well as $1 \mathrm{~h}$ prior to the procedure. Evans Blue $(4 \mathrm{mg} / \mathrm{kg})$ was intravenously injected immediately following photothrombotic ischemic insult. (a) Representative photographs of Evans Blue leakage in control or Shunaghe-tang groups $24 \mathrm{~h}$ after ischemic injury. (b) Quantification of Evans Blue extravasation. ${ }^{*} p<0.05$ versus control group.

diseases due to its proven anti-inflammatory, antiulcer, and renoprotective therapeutic potentials [26].

Ancient Korean medical practitioners developed prescriptions using various combinations of medicinal herbs. These combinations of herbs were believed to enhance the therapeutic effect of treatment and reduce toxicity [27]. The medicinal herbs of such prescriptions were classified as follows: Emperor, which was used to refer to the herb with the highest component ratio within the prescription; Minister, which referred to herbs purported to enhance treatment effects; Assistant, which referred to herbs used to reduce the side effects of other ingredients; and Courier, which referred to herbs used to target the desired organ or balance the therapeutic effects of other components [28]. In the present study, we selected two such prescriptions-Shuanghetang and Zengsunsiwu-tang (Table 2)-based on the following principles: (1) The candidate herbs are the main medicinal herbs within the prescription (Emperor), (2) the formula includes at least four of the five candidate herbs, and (3) the volume of these four herbs comprises over $60 \%$ of the total volume of the combined formula. Shuanghe-tang has long been utilized to treat fatigue and promote recuperation following sickness in Korea [29]. According to the Dongui Bogam, Zengsunsiwu-tang has been used in the treatment of continuous bleeding after giving birth [29].

In the present study, mice were pretreated with either Shuanghe-tang or Zengsunsiwu-tang prior to ischemic brain injury (Figures 3 and 4). Our findings indicated that Shuanghe-tang treatment significantly reduced direct infarct and edema volume, in addition to increasing neurological, locomotor, and vestibular function. Such findings indicate that Shuanghe-tang exerts protective effects against ischemic brain damage. In contrast, treatment with Zengsunsiwu-tang produced no significant reductions in the extent or functional impact of ischemic injury.

The $\mathrm{BBB}$ is maintained by tight junction proteins (e.g., ZO-1, occludin, and claudin-5) between endothelial cells, astrocyte endfeet, and pericytes [30]. Due to its structure, the $\mathrm{BBB}$ plays an important role in maintaining the appropriate concentrations of ions such as $\mathrm{Na}^{+}, \mathrm{K}^{+}$, and $\mathrm{Ca}^{2+}$ within a narrow space [31]. Following ischemic stroke, the integrity of the $\mathrm{BBB}$ is compromised, and the resulting increase in permeability enables the infiltration of circulating immune cells into the brain [13]. Such infiltration aggravates inflammation and generates neurotoxicity at the damaged site, thereby resulting in neuronal cell death [32]. Therefore, prevention of BBB breakdown represents a major strategy for the attenuation of brain damage following ischemic injury. The results of the present study indicate that Shuanghe-tang pretreatment reduces BBB disruption and increases levels of occludin and $\mathrm{ZO}-1$ in the ischemic brain (Figures 5 and 6).

Alterations in AQP4, a water channel protein that regulates water homeostasis, play an important role in ischemiainduced changes in BBB integrity [16]. It was also reported that AQP4 is associated with neuroinflammation in brain diseases [33, 34]. We observed that downregulated AQP4 in the peri-infarct region was significantly increased in mice pretreated with Shuanghe-tang (Figure 7). We also examined microglia activation in the peri-infarct region using Iba-1 (microglia marker protein) by immunohistochemical staining. But no significant change for Iba-1 was observed in the cortex of Shuanghe-tang pretreatment group relative to the control group (data not shown). These data suggest that neuroinflammation such as microglia activation after ischemic injury was not affect by Shuanghe-tang pretreatment. The effects of AQP4 on BBB injury and cerebral edema remain controversial. Several studies have indicated that AQP4 expression is upregulated in the mouse model of stroke and brain edema could be moderated by downregulation of AQP4 [19,35]. Conversely, reduction of AQP4 aggravates edema size $[18,36]$ and Apelin-13 protects against ischemic vascular leakage via AQP4 induction [17]. Taken together, these findings indicate that Shuanghe-tang pretreatment may attenuate BBB dysfunction and edema following ischemic brain injury by promoting increased expression of ZO-1, occludin, and AQP4. However, further studies are required to investigate the mechanisms underlying the effects of Shuanghe-tang on the expression of tight junction proteins and AQP4. 


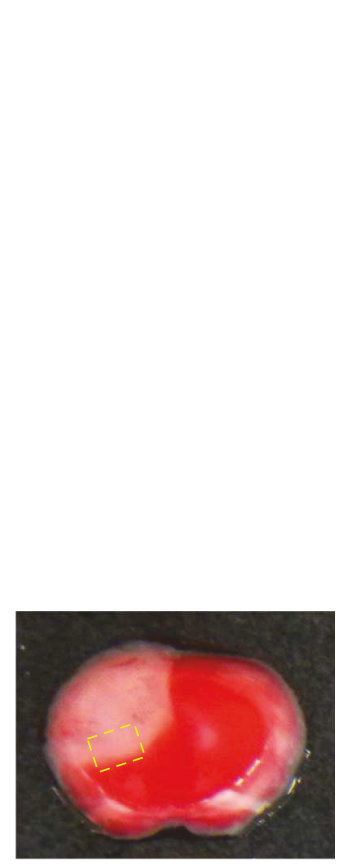

(a)

CD-31
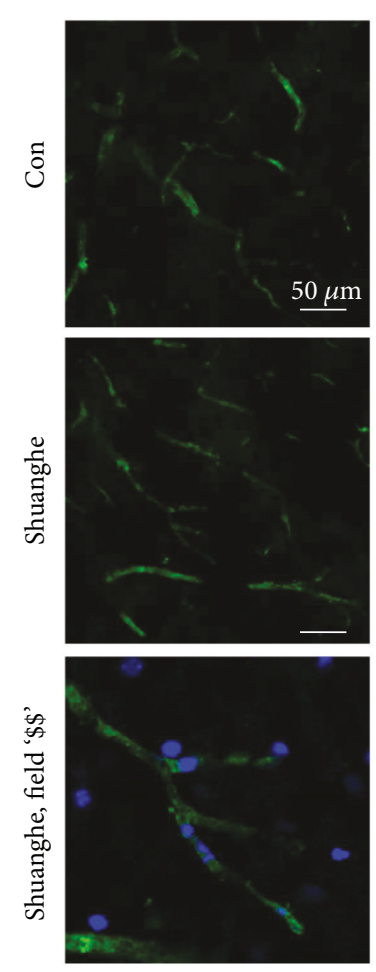

ZO-1

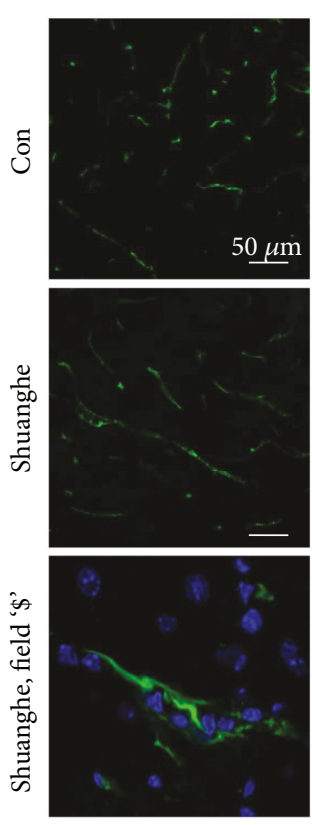

Occludin
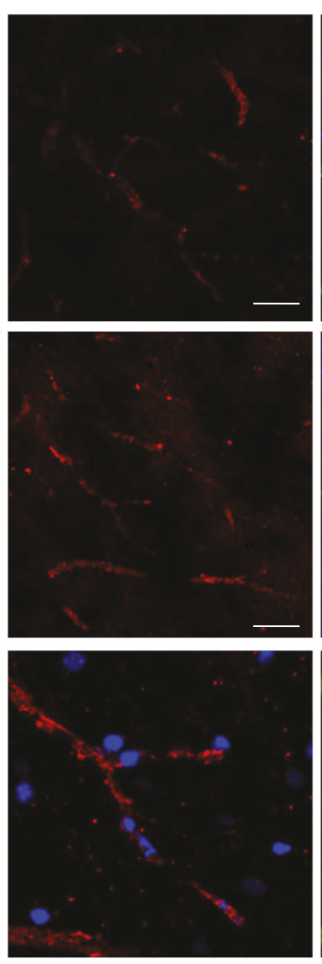

(d)

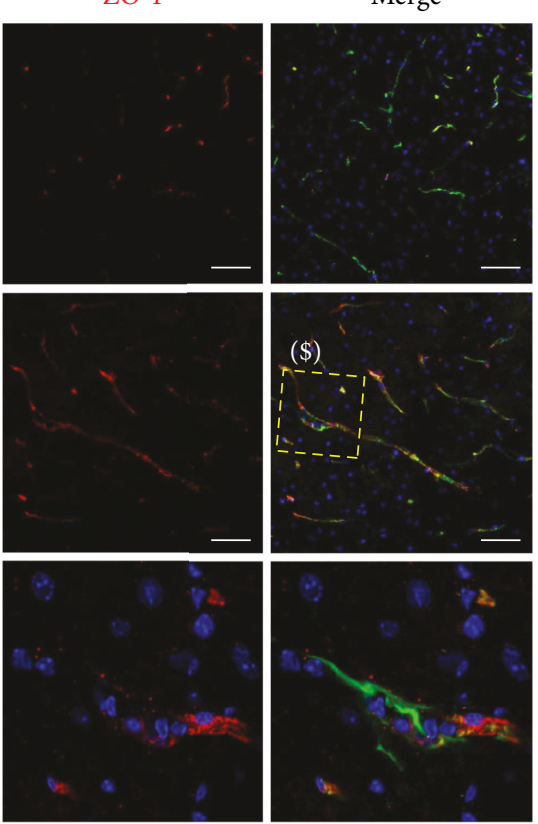

(b)

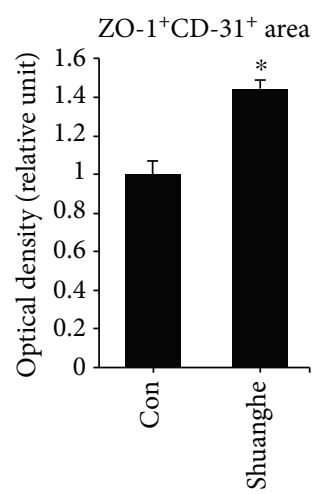

(c)
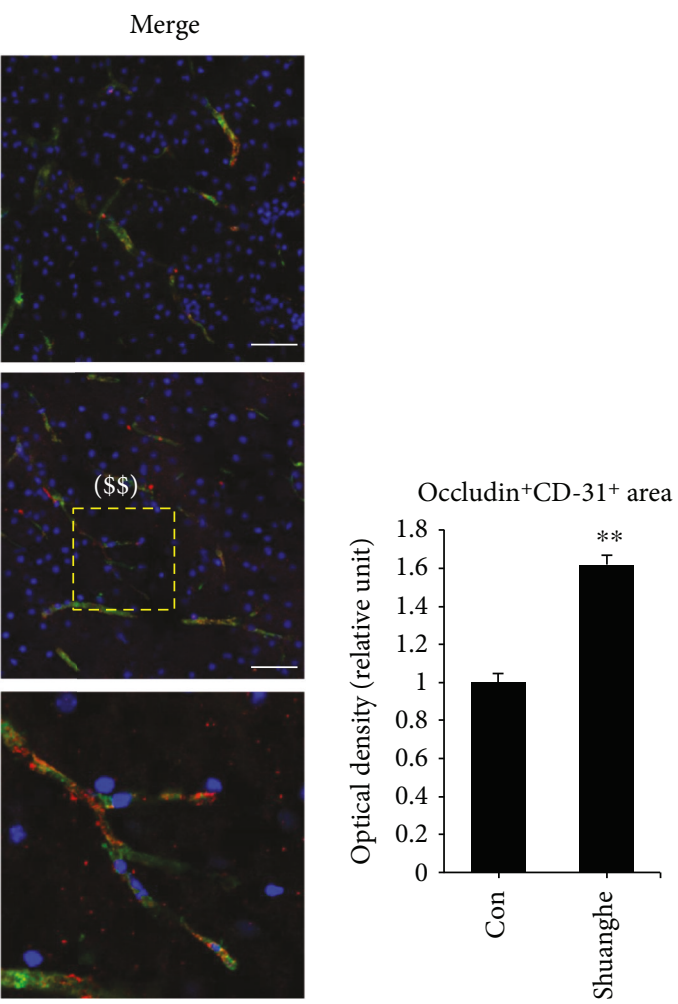

(e)

FIGURE 6: Effects of Shuanghe-tang pretreatment on ZO-1 and occludin levels in ischemic mouse brains. Mice pretreated with Shuanghetang $(300 \mathrm{mg} / \mathrm{kg})$ exhibited increased expression of the tight junction proteins ZO-1 (b, c) and occludin (d, e) following focal cerebral ischemia. (a) Dashed yellow square indicates the photographed area. Representative photographs of ZO-1 (\$) and occludin (\$). CD-31 staining for blood vessels is indicated in green. Scale bar $=50 \mu \mathrm{m}$. Quantification graphs of ZO-1 (c) and occludin (e) immunofluorescence ( $n=6$ each, ${ }^{*} p<0.05,{ }^{* *} p<0.01$ versus control group). 

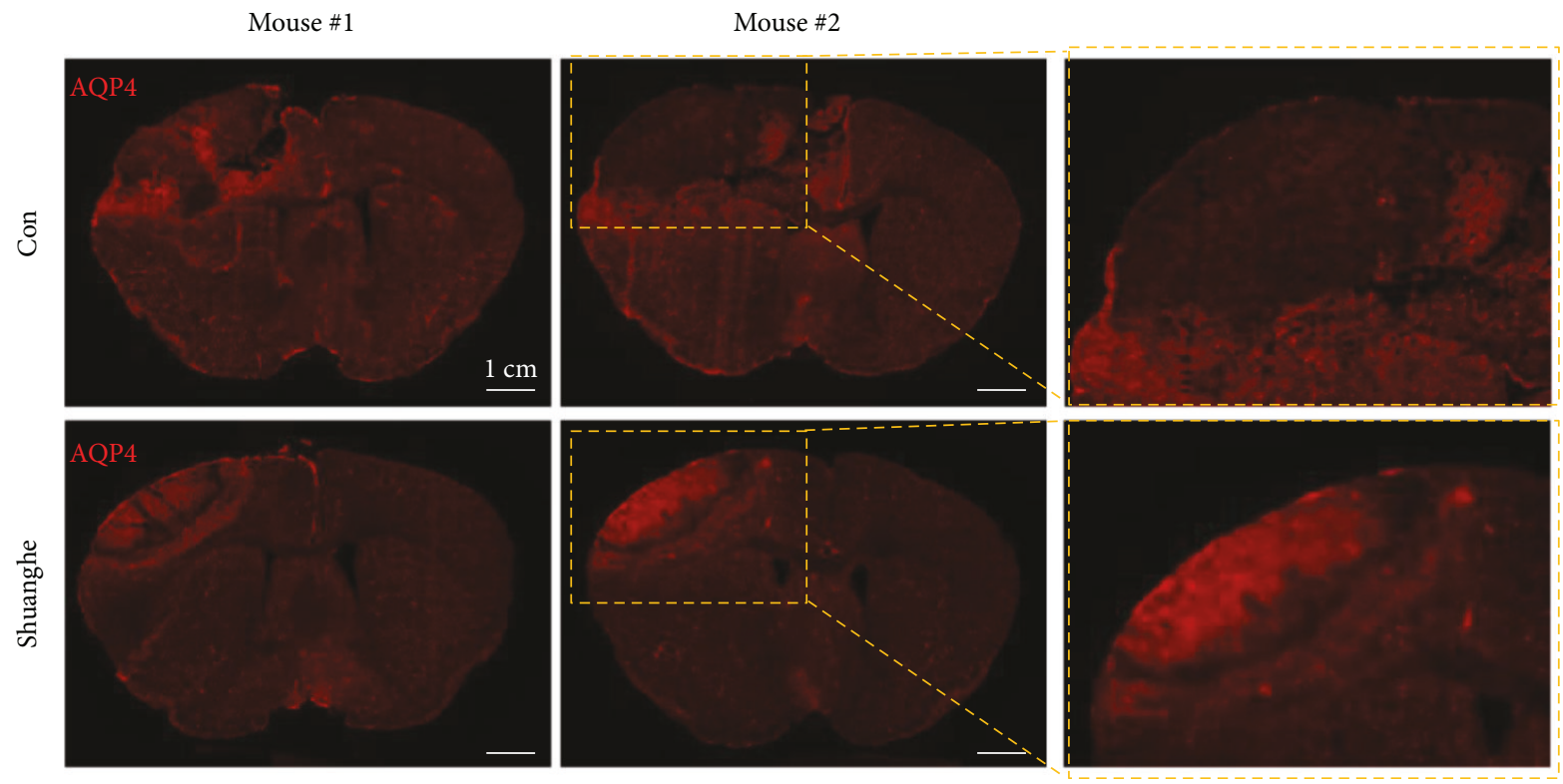

(a)
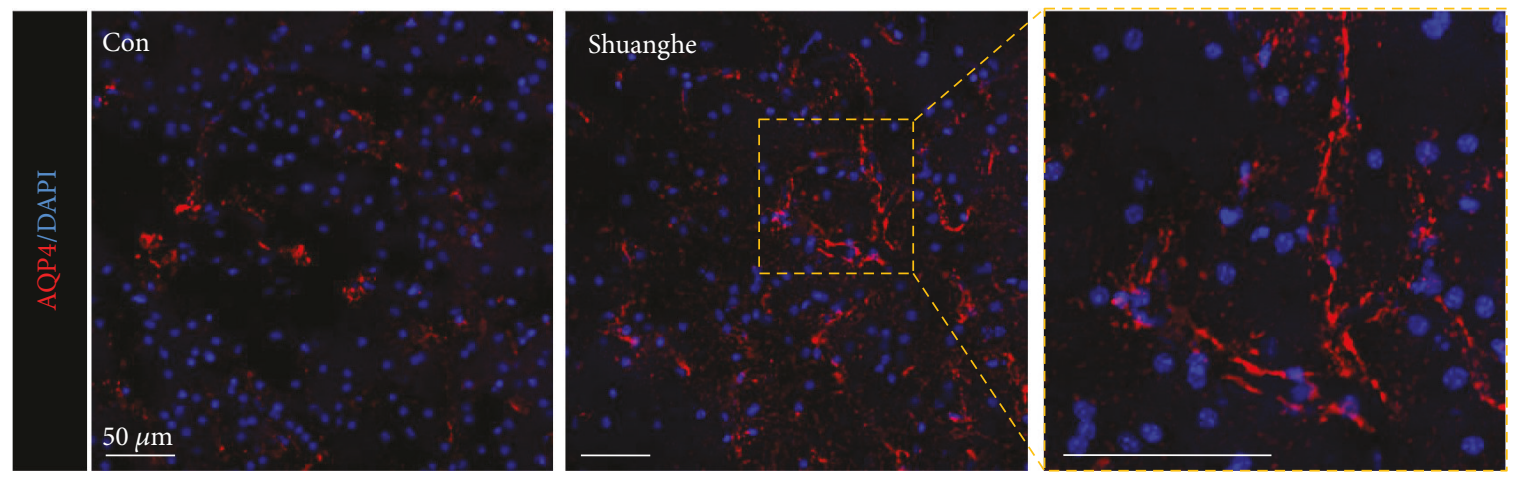

(b)

$\mathrm{AQP}^{+}$area

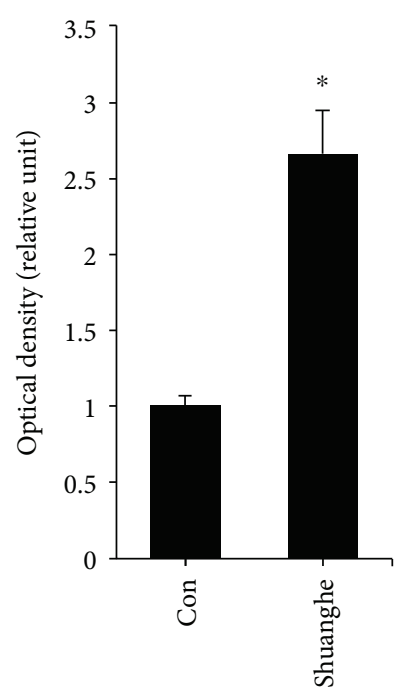

(c)

Figure 7: Continued. 

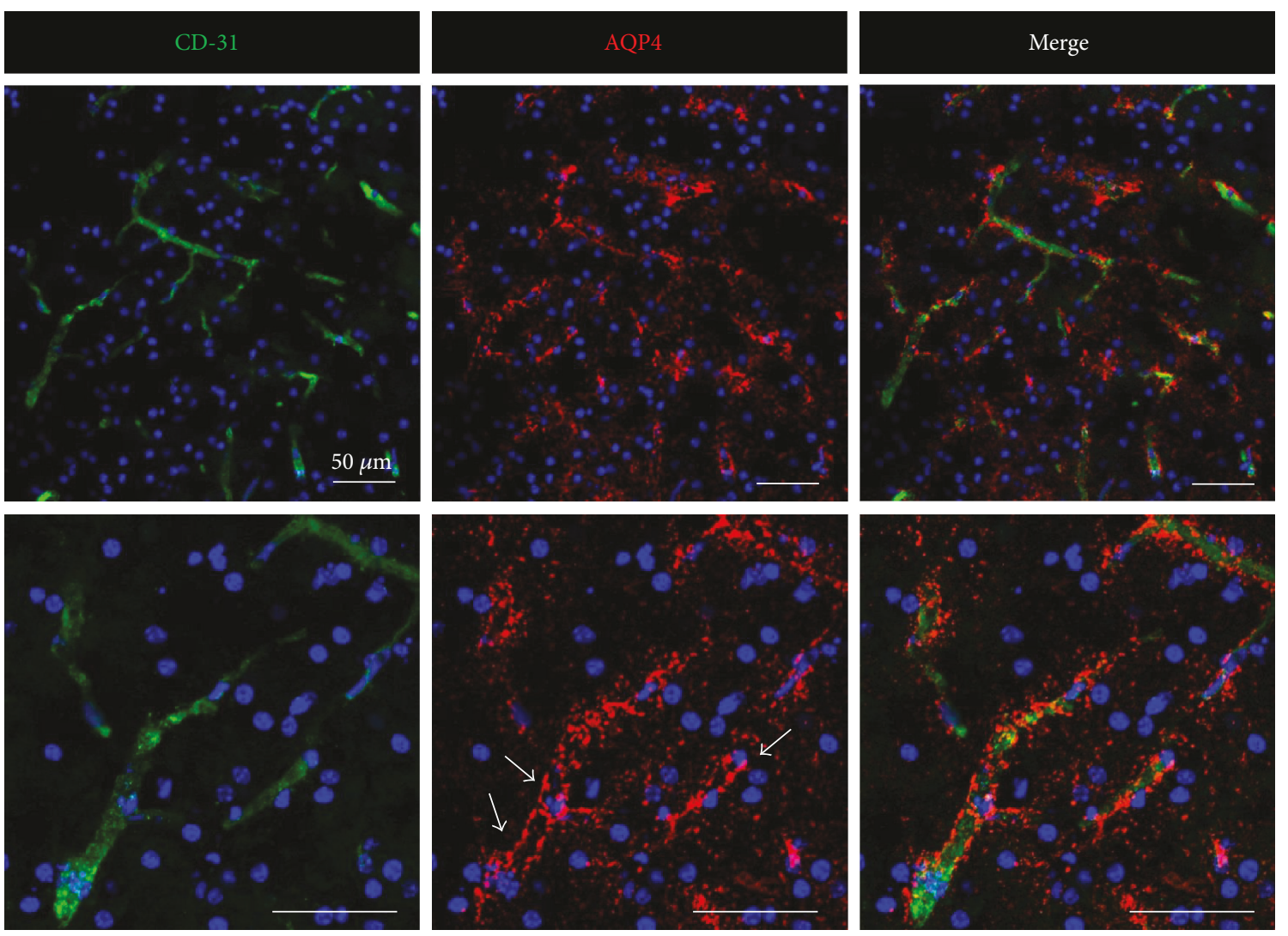

(d)

FIGURE 7: Changes in AQP-4 levels in ischemic mouse brains. (a, b) AQP4 immunofluorescence levels were low in the ischemic area, although such reductions were increased in mice pretreated with Shuanghe-tang. (a) Magnification $=\times 10$; Scale bar $=1 \mathrm{~cm}$. (b) magnification $=\times 200$. Scale bar $=50 \mu \mathrm{m}$. (c) Quantification of AQP4 ${ }^{+}$area. $(n=3) .{ }^{*} p<0.05$ versus control group. (d) The ipsilateral cerebral cortex of mice pretreated with Shuanghe-tang. AQP4 (red, arrows) restricted to astrocytic endfeet contacts the blood vessel (green CD-31). Scale bar $=50 \mu \mathrm{m}$.

\section{Conclusion}

In the present study, we identified a therapeutic formula for ischemic stroke based on candidate herbs selected via text mining of a traditional Korean medical text (Dongui Bogam). Further analysis in a mouse model of photothrombotic stroke indicated that Shuanghe-tang exerts protective effects against ischemic brain damage and promotes recovery of neurological and motor function following focal cerebral ischemia. Further experimental and clinical investigations of Shuanghe-tang may aid in the development of novel therapeutic strategies for ischemic stroke.

\section{Conflicts of Interest}

The authors declare that they have no competing interests.

\section{Authors' Contributions}

Min Jae Kim, Seo-Yeon Lee, Byung Tae Choi, Jin Ung Baek, and Hwa Kyoung Shin participated in the research design. Min Jae Kim, Ji Young Hwang, and Hyunha Kim conducted the experiments. Ki-Tae Ha and Jin Ung Baek contributed new reagents or analytic tools. Min Jae Kim, Seo-Yeon Lee, and Hwa Kyoung Shin performed data analysis. Min Jae Kim, Seo-Yeon Lee, Byung Tae Choi, Jin Ung Baek, and Hwa Kyoung Shin wrote or contributed to the writing of the manuscript. Min Jae Kim and Seo-Yeon Lee contributed equally to this work.

\section{Acknowledgments}

This work was supported by the National Research Foundation of Korea (NRF) grant funded by the Korea government (MSIP) (2014R1A5A2009936).

\section{Supplementary Materials}

Figure S1: preparation of ethanol extracts (A) and water extracts (B). Figure S2: posttreatment effects of Shuanghetang on brain function and behavior in brain injury. (A) After focal ischemic injury, mice underwent oral administration of Shuanghe-tang $(300 \mathrm{mg} / \mathrm{kg}, n=7)$ or PBS (control group, $n=5$ ) once per day for 3 days. Twenty-four hours after final administration, the mouse brains were harvested and stained with 2\% TTC solution. (B) Representative photographs of brain sections stained with TTC. White region (arrows) indicates the infarct area. (C, D) Quantification of 
direct infarct volume (C) and edema (D). (E-G) Neurological score $(\mathrm{E})$, rota-rod $(\mathrm{F})$, and wire grip $(\mathrm{G})$ were evaluated to assess recovery of neurologic deficit, locomotor function, and vestibular motor function after ischemic injury. (Supplementary Materials)

\section{References}

[1] G. A. Donnan, M. Fisher, M. Macleod, and S. M. Davis, "Stroke," Lancet, vol. 371, no. 9624, pp. 1612-1623, 2008.

[2] J. J. Guilbert, "The world health report 2002 - reducing risks, promoting healthy life," Education for Health: Change in Learning \& Practice, vol. 16, no. 2, p. 230, 2003.

[3] B. S. Alper, M. Malone-Moses, J. S. McLellan, K. Prasad, and E. Manheimer, "Thrombolysis in acute ischaemic stroke: time for a rethink?," BMJ, vol. 350, no. mar17 3, p. h1075, 2015.

[4] D. X. Kong, X. J. Li, and H. Y. Zhang, "Where is the hope for drug discovery? Let history tell the future," Drug Discovery Today, vol. 14, no. 3-4, pp. 115-119, 2009.

[5] F. E. Koehn and G. T. Carter, "The evolving role of natural products in drug discovery," Nature Reviews. Drug Discovery, vol. 4, no. 3, pp. 206-220, 2005.

[6] B. H. May, C. Lu, L. Bennett, H. M. Hugel, and C. C. Xue, "Evaluating the traditional Chinese literature for herbal formulae and individual herbs used for age-related dementia and memory impairment," Biogerontology, vol. 13, no. 3, pp. 299-312, 2012.

[7] M. J. Choi, B. T. Choi, H. K. Shin, B. C. Shin, Y. K. Han, and J. U. Baek, "Establishment of a comprehensive list of candidate antiaging medicinal herb used in Korean medicine by text mining of the classical Korean medical literature, "dongeuibogam," and preliminary evaluation of the antiaging effects of these herbs," Evidence-based Complementary and Alternative Medicine, vol. 2015, Article ID 873185, 2015.

[8] M. Y. Park, Y. S. Jung, J. H. Park et al., "PMC-12, a prescription of traditional Korean medicine, improves amyloid betainduced cognitive deficits through modulation of neuroinflammation," Evidence-based Complementary and Alternative Medicine, vol. 2015, Article ID 768049, 2015.

[9] B. C. Yun, S. B. Pae, Y. K. Han et al., "An analysis of the combination frequencies of constituent medicinal herbs in prescriptions for the treatment of stroke in Korean medicine: determination of a group of candidate prescriptions for universal use," Evidence-based Complementary and Alternative Medicine, vol. 2016, Article ID 2674014, 17 pages, 2016.

[10] T. M. Woodruff, J. Thundyil, S. C. Tang, C. G. Sobey, S. M. Taylor, and T. V. Arumugam, "Pathophysiology, treatment, and animal and cellular models of human ischemic stroke," Molecular Neurodegeneration, vol. 6, no. 1, p. 11, 2011.

[11] J. D. Huber, R. D. Egleton, and T. P. Davis, "Molecular physiology and pathophysiology of tight junctions in the bloodbrain barrier," Trends in Neurosciences, vol. 24, no. 12, pp. 719-725, 2001.

[12] S. W. Lee, W. J. Kim, Y. K. Choi et al., "SSeCKS regulates angiogenesis and tight junction formation in blood-brain barrier," Nature Medicine, vol. 9, no. 7, pp. 900-906, 2003.

[13] Y. Shi, L. Zhang, H. Pu et al., "Rapid endothelial cytoskeletal reorganization enables early blood-brain barrier disruption and long-term ischaemic reperfusion brain injury," Nature Communications, vol. 7, p. 10523, 2016.
[14] D. Toni, M. Fiorelli, M. Gentile et al., "Progressing neurological deficit secondary to acute ischemic stroke. A study on predictability, pathogenesis, and prognosis," Archives of Neurology, vol. 52, no. 7, pp. 670-675, 1995.

[15] J. Kirk, J. Plumb, M. Mirakhur, and S. McQuaid, "Tight junctional abnormality in multiple sclerosis white matter affects all calibres of vessel and is associated with blood-brain barrier leakage and active demyelination," The Journal of Pathology, vol. 201, no. 2, pp. 319-327, 2003.

[16] H. Chu, C. Huang, H. Ding et al., "Aquaporin-4 and cerebrovascular diseases," International Journal of Molecular Sciences, vol. 17, no. 8, 2016.

[17] H. Chu, X. Yang, C. Huang, Z. Gao, Y. Tang, and Q. Dong, "Apelin-13 protects against ischemic blood-brain barrier damage through the effects of aquaporin-4," Cerebrovascular Diseases, vol. 44, no. 1-2, pp. 10-25, 2017.

[18] L. Hirt, B. Ternon, M. Price, N. Mastour, J. F. Brunet, and J. Badaut, "Protective role of early aquaporin 4 induction against postischemic edema formation," Journal of Cerebral Blood Flow and Metabolism, vol. 29, no. 2, pp. 423-433, 2008.

[19] L. Hirt, A. M. Fukuda, K. Ambadipudi et al., "Improved longterm outcome after transient cerebral ischemia in aquaporin-4 knockout mice," Journal of Cerebral Blood Flow and Metabolism, vol. 37, no. 1, pp. 277-290, 2016.

[20] H. I. Lee, S. W. Lee, N. G. Kim et al., "Low-level light emitting diode (LED) therapy suppresses inflammasome-mediated brain damage in experimental ischemic stroke," Journal of Biophotonics, vol. 10, no. 11, pp. 1502-1513, 2017.

[21] X. Li, K. K. Blizzard, Z. Zeng, A. C. DeVries, P. D. Hurn, and L. D. McCullough, "Chronic behavioral testing after focal ischemia in the mouse: functional recovery and the effects of gender," Experimental Neurology, vol. 187, no. 1, pp. 94-104, 2004.

[22] H. B. Seo, B. K. Kang, J. H. Kim et al., "Partially purified components of Uncaria sinensis attenuate blood brain barrier disruption after ischemic brain injury in mice," $B M C$ Complementary and Alternative Medicine, vol. 15, no. 1, p. $157,2015$.

[23] D. W. Lim, C. Lee, I. H. Kim, and Y. T. Kim, “Anti-inflammatory effects of total isoflavones from Pueraria lobata on cerebral ischemia in rats," Molecules, vol. 18, no. 9, pp. 10404-10412, 2013.

[24] S. H. Son, M. J. Kim, W. Y. Chung et al., "Decursin and decursinol inhibit VEGF-induced angiogenesis by blocking the activation of extracellular signal-regulated kinase and c-Jun N-terminal kinase," Cancer Letters, vol. 280, no. 1, pp. 86-92, 2009.

[25] S. H. Lee, J. H. Lee, E. Y. Oh et al., "Ethanol extract of Cnidium officinale exhibits anti-inflammatory effects in BV2 microglial cells by suppressing NF- $\kappa \mathrm{B}$ nuclear translocation and the activation of the PI3K/Akt signaling pathway," International Journal of Molecular Medicine, vol. 32, no. 4, pp. 876-882, 2013.

[26] S. Ojha, M. Golechha, S. Kumari, J. Bhatia, and D. S. Arya, "Glycyrrhiza Glabra protects from myocardial ischemiareperfusion injury by improving hemodynamic, biochemical, histopathological and ventricular function," Experimental and Toxicologic Pathology, vol. 65, no. 1-2, pp. 219-227, 2013.

[27] W. Jia, W. Y. Gao, Y. Q. Yan et al., "The rediscovery of ancient Chinese herbal formulas," Phytotherapy Research, vol. 18, no. 8, pp. 681-686, 2004. 
[28] X. Zhou, S. W. Seto, D. Chang et al., "Synergistic effects of Chinese herbal medicine: a comprehensive review of methodology and current research," Frontiers in Pharmacology, vol. 7, p. 201, 2016

[29] A. Kim, N. H. Yim, M. Im et al., "Ssanghwa-tang, an oriental herbal cocktail, exerts anti-melanogenic activity by suppression of the p38 MAPK and PKA signaling pathways in B16F10 cells," BMC Complementary and Alternative Medicine, vol. 13, no. 1, p. 214, 2013.

[30] E. A. Neuwelt, B. Bauer, C. Fahlke et al., "Engaging neuroscience to advance translational research in brain barrier biology," Nature Reviews. Neuroscience, vol. 12, no. 3, pp. 169-182, 2011.

[31] B. T. Hawkins and T. P. Davis, "The blood-brain barrier/neurovascular unit in health and disease," Pharmacological Reviews, vol. 57, no. 2, pp. 173-185, 2005.

[32] C. V. Borlongan, L. E. Glover, P. R. Sanberg, and D. C. Hess, "Permeating the blood brain barrier and abrogating the inflammation in stroke: implications for stroke therapy," Current Pharmaceutical Design, vol. 18, no. 25, pp. 3670-3676, 2012.

[33] A. M. Fukuda and J. Badaut, "Aquaporin 4: a player in cerebral edema and neuroinflammation," Journal of Neuroinflammation, vol. 9, no. 1, p. 279, 2012.

[34] J. Badaut, A. M. Fukuda, A. Jullienne, and K. G. Petry, "Aquaporin and brain diseases," Biochimica et Biophysica Acta, vol. 1840, no. 5, pp. 1554-1565, 2014.

[35] G. T. Manley, M. Fujimura, T. Ma et al., "Aquaporin-4 deletion in mice reduces brain edema after acute water intoxication and ischemic stroke," Nature Medicine, vol. 6, no. 2, pp. 159-163, 2000.

[36] S. Meng, M. Qiao, L. Lin, M. R. Del Bigio, B. Tomanek, and U. I. Tuor, "Correspondence of AQP4 expression and hypoxic-ischaemic brain oedema monitored by magnetic resonance imaging in the immature and juvenile rat," The European Journal of Neuroscience, vol. 19, no. 8, pp. 22612269, 2004. 


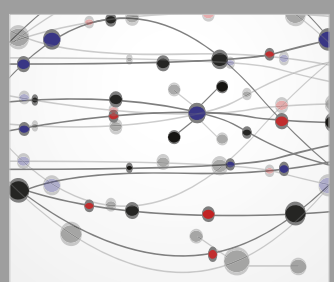

The Scientific World Journal
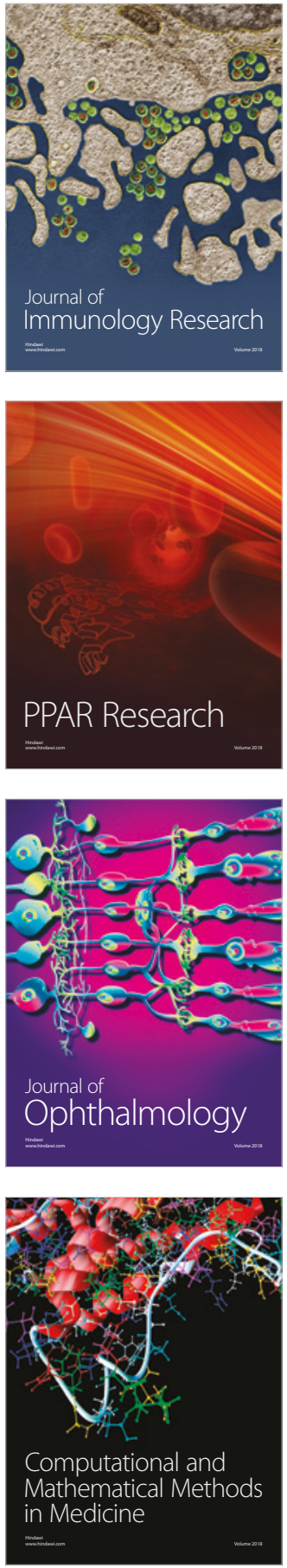

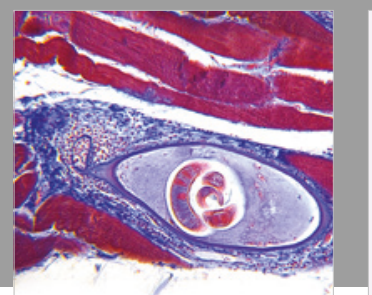

Gastroenterology Research and Practice

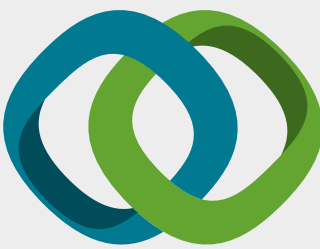

\section{Hindawi}

Submit your manuscripts at

www.hindawi.com
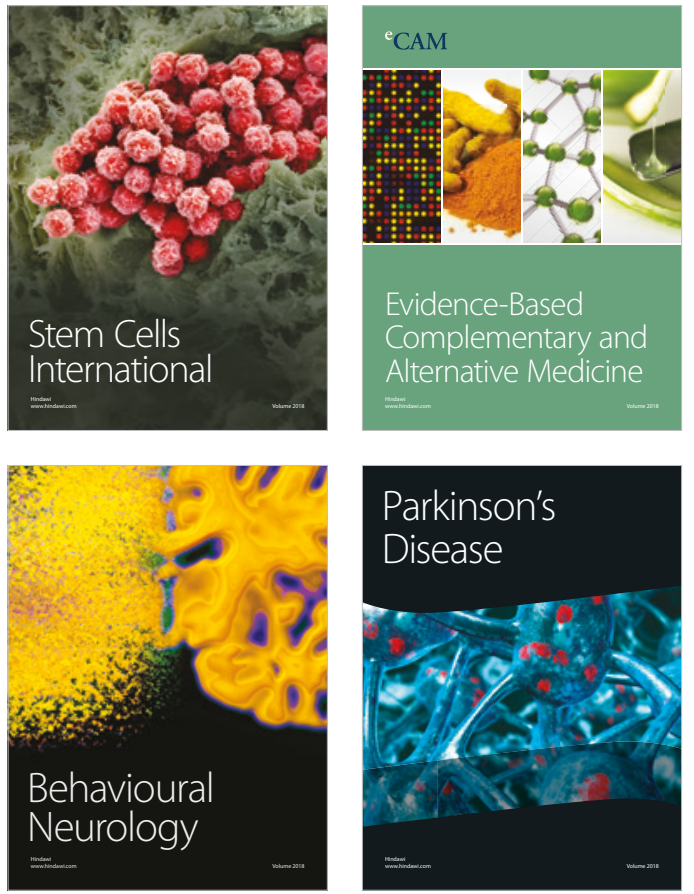

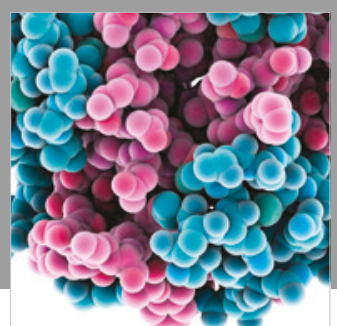

ournal of

Diabetes Research

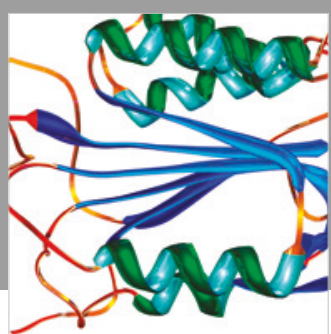

Disease Markers
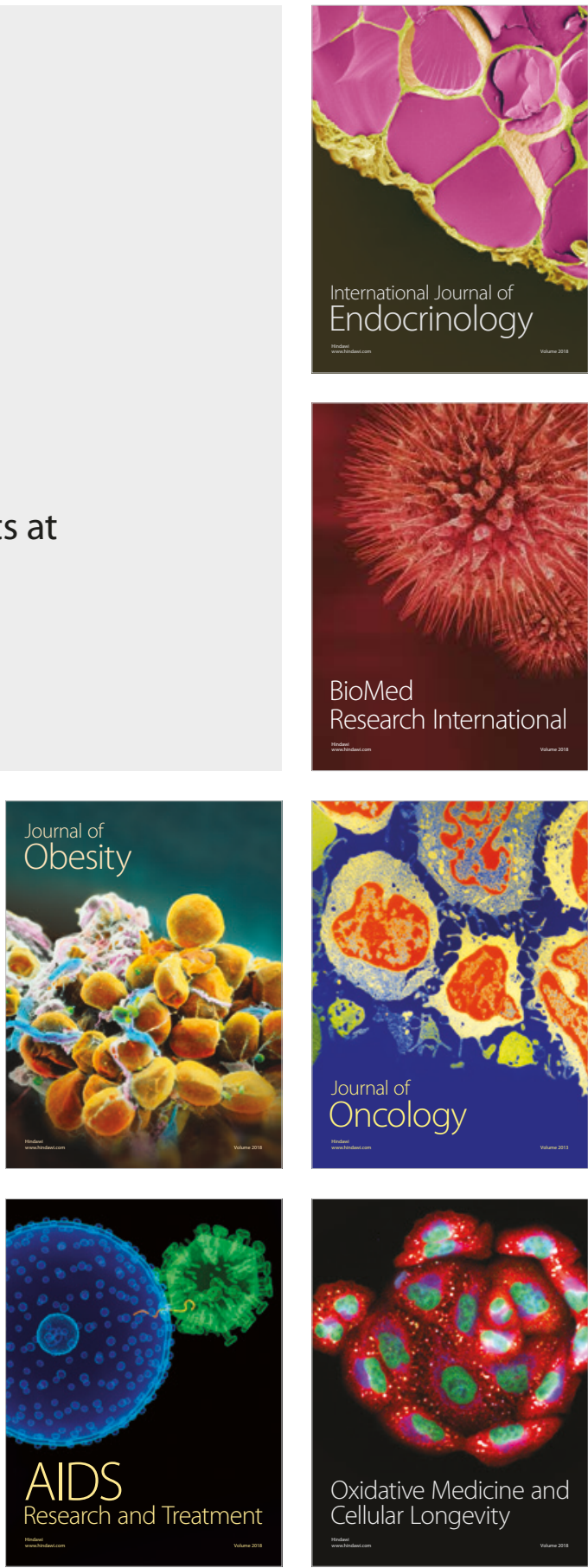